\title{
Anorectal melanoma and gene analysis of personalized adjuvant therapy: a case report
}

\author{
Xiaochen Cai, Xiaoming Zhu \\ Department of Gastroenterological Surgery, Yueyang Hospital, Shanghai University of Traditional Chinese Medicine, Shanghai, China \\ Correspondence to: Xiaoming Zhu. Department of Gastroenterological Surgery, Yueyang Hospital, Shanghai University of Traditional Chinese \\ Medicine, 110 Ganhe Road, Shanghai 200437, China. Email: miller_zxm@163.com.
}

\begin{abstract}
Melanoma is derived from malignancies of melanocytes. Anorectal melanoma differs significantly from cutaneous melanoma in clinical presentation, genetic profile, staging system, and response to treatment. Anorectal melanoma is seldom diagnosed because most melanoma occurrences are found in the skin tissues. Here, we report 1 case of advanced anorectal melanoma, including its clinical presentation, laboratory findings, imaging, surgical treatment, and pathology. The patient complained of hematochezia and tenesmus. Colonoscopy, computed tomography (CT) scan and digital rectal examination (DRE) revealed a mass near the pectinate line. The patient underwent proctectomy along with colostomy, and subsequent pathological examinations suggested anorectal melanoma with serosa involvement (positive markers: S100, HMB-45, etc.). Evidence-based analyses (single-nucleotide polymorphism (SNPs) and programmed death-ligand 1 (PD-L1) expression) were conducted on the tumor tissue to identify the sensitivity to adjuvant therapies. SNP tests suggested no definite efficacies of commonly used chemotherapeutic agents, with PD-L1 expression implying poor sensitivity to PD-L1 inhibitors. The postoperative recovery was uneventful and the patient was discharged on day 7 after admission. However, the patient refused adjuvant therapies and died 11 months after surgery. In conclusion, anorectal melanoma tends to be mistaken for other common diseases in this region owing to its non-specific clinical presentations. Multidisciplinary treatments are recommended to yield the best possible outcome, despite poor prognosis.
\end{abstract}

Keywords: Anorectal melanoma; adjuvant therapy; case report

Submitted Jul 16, 2021. Accepted for publication Oct 14, 2021.

doi: 10.21037/apm-21-2240

View this article at: https://dx.doi.org/10.21037/apm-21-2240

\section{Introduction}

Anorectal melanoma is a rare subtype of malignancy that accounts for a very little percentage in both rectal malignancy (in which rectal adenocarcinoma is the most common) and melanoma (in which skin melanoma is the most common). While some localized cases can be cured by surgical resection, overall survival rates are discouraging compared with those of cutaneous melanoma because anorectal melanoma can develop occultly until obvious symptoms occur (1). Optimal treatment strategies are yet to be found to extend the poor overall survival rate. We present the following article in accordance with the CARE reporting checklist (available at https://dx.doi.org/10.21037/apm-21-2240).

\section{Case presentations}

Our patient was an 81-year-old male, who complained of hematochezia and tenesmus for approximately 1 month. The patient was admitted due to digital rectal examination (DRE) findings of a rectal mass $2 \mathrm{~cm}$ from the anus, with visible blood. The patient denied any medical history or relevant past interventions. Further colonoscopy revealed a $5 \mathrm{~cm} \times 4 \mathrm{~cm}$ mass near the pectinate line, with ulceration on the surface (Figure 1), which was confirmed by pelvic computed tomography (CT) scan (Figure 2). Abdominal CT scan suggested multiple metastases on the liver (Figure 3). Blood test indicated no sign of anemia, and serum tumor markers [e.g., carcinoembryonic antigen 


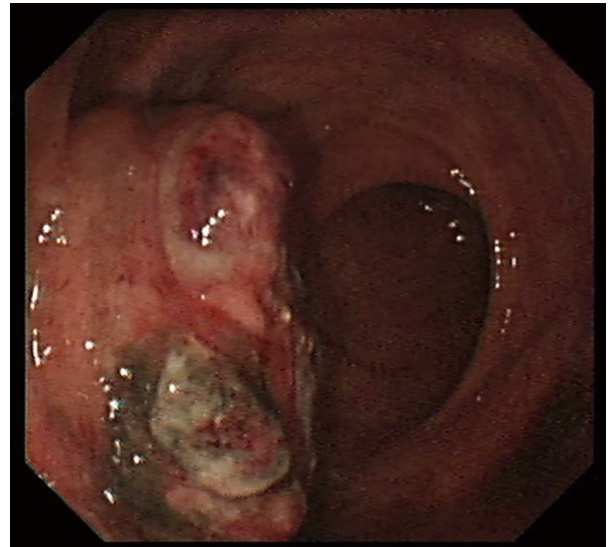

Figure 1 Coloscopy image of the rectal melanoma.

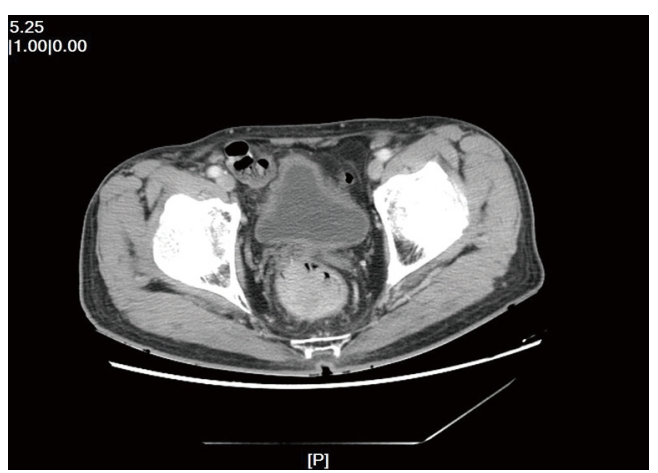

Figure 2 Cross-sectional image of the tumor from a pelvic computed tomography scan.

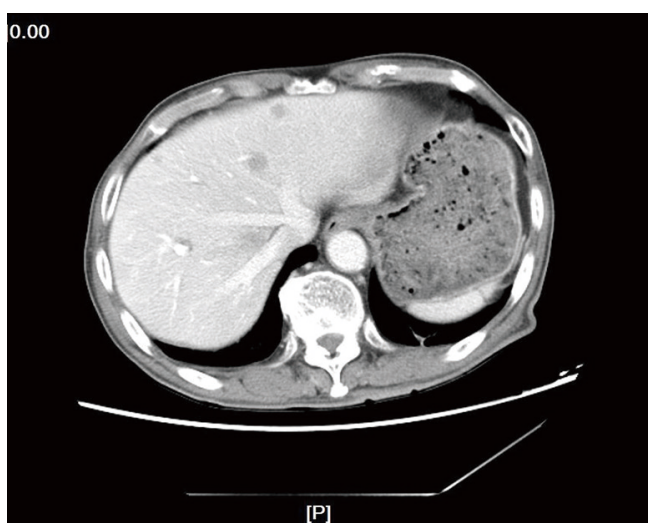

Figure 3 Cross-sectional image of multiple metastases on the liver from an abdominal computed tomography scan.
(CEA), carbohydrate antigen 19-9 (CA19-9), CA125] for gastrointestinal cancers were within normal range; an elevated lactate dehydrogenase $(\mathrm{LDH})$ level $(260 \mu / \mathrm{L})$ was detected. Biopsy via colonoscopy suggested the primary tumor was of non-epithelial malignant origin. All procedures performed in studies involving human participants were in accordance with the ethical standards of the institutional and/or national research committee(s) and with the Helsinki Declaration (as revised in 2013). Written informed consent was obtained from the patient for publication of this manuscript and any accompanying images. A copy of the written consent is available for review by the editorial office of this journal.

\section{Treatment and patbology}

After providing signed informed consent, the patient underwent proctectomy along with colostomy, and subsequent pathological examinations were carried out (Table 1). Pathological staging was determined as stage III (1).

\section{Adjuvant therapy sensitivity analyses}

Single-nucleotide polymorphism (SNPs) were tested with regard to efficacies of commonly used chemotherapeutic agents according to the PharmGKB database (Table 2). SNP tests suggested no definite efficacy of chemotherapy. CD274 (programmed death-ligand 1 or PD-L1) expression was not detected via immunohistochemical staining (Figure 4A,4B), which implied poor sensitivity to PD-L1 inhibitors.

\section{Follow up and outcome}

The postoperative recovery was uneventful, and the patient received proper training on the management of the stoma. However, the patient refused adjuvant therapies and followup examinations, and died 11 months after surgery. The timeline of the case is shown in Table 3.

\section{Discussion}

The typical primary location for melanoma is the skin; on the legs for female and on the back for males (2). 
Table 1 Pathology report summary

\begin{tabular}{ll}
\hline Variable & Outcome \\
\hline Tumor size $(\mathrm{cm})$ & $5 \times 4.5 \times 3$ \\
Tumor type & Melanoma \\
Tumor depth & Serosa involvement present \\
Margin involvement & Absent \\
Surrounding tissue & Absent \\
involvement & \\
Lymphatic invasion & Absent \\
Vascular invasion & Absent \\
Perineural invasion & Absent \\
Immunohistochemistry & \\
Positive & S100, HMB-45, CD45R0 (partially), \\
& CD31 (on vessels), CD34 (on \\
& vessels), Ki67 (30\%) \\
Weakly positive & Vimentin, CD20, CD3, CD79a, \\
CD117, P53 & CK, Bcl-6, Dog-1, SMA \\
\hline
\end{tabular}

Bcl-6, B-cell Lymphoma 6; CK, cytokeratin; Dog-1, discovered on GIST 1; SMA, smooth muscle actin.
Less frequent primary locations include the uveal and anorectal region, with anorectal melanoma accounting for less than $5 \%$ of all anorectal malignancies and less than $2 \%$ of all primary melanomas (3). Anorectal melanoma differs significantly from cutaneous melanoma in clinical presentation, genetic profile, staging system, and response to treatment (4). Therefore, the National Comprehensive Cancer Network (NCCN) guideline for melanoma currently does not include anorectal melanoma. A consensus staging system is yet to be established, but in some studies, anorectal melanomas were classified as stage I (local disease), stage II (regional metastasis), or stage III (distant metastasis) (1,5), which was applied in our case. The 5-year survival rate for cases with distant metastasis is extremely poor (nearly $0 \%$ ) (1). In our case, the patient was found to have liver metastases on admission, which was indicative of poor prognosis.

Anorectal melanoma tends to be mistaken for other common diseases in this region owing to its non-specific clinical presentations, such as hematochezia, perianal mass, pruritus, tenesmus, and prolapsed hemorrhoid (6). With appropriate biomarkers yet to be found, early diagnose of anorectal melanoma depends predominantly on timely

Table 2 SNPs regarding chemotherapy efficacies

\begin{tabular}{|c|c|c|c|c|c|}
\hline Chemotherapeutic agents & Gene & Position & Genotype & Evidence level & Implication \\
\hline \multirow[t]{7}{*}{ Platinum based } & ERCC1 & rs11615 & $\mathrm{CT}$ & 2B & Decreased efficacy \\
\hline & ERCC2 & rs11615 & AA (Lys/Lys) & 3 & Increased efficacy \\
\hline & GSTP1 & rs1695 & AA (lle/lle) & $2 \mathrm{~A}$ & Decreased efficacy \\
\hline & $X R C C 1$ & rs25487 & GG (Arg/Arg) & $2 B$ & Increased efficacy \\
\hline & TPMT & rs1800462 & GG & 3 & Reduced side-effects \\
\hline & & rs1800460 & GG & & \\
\hline & & rs1142345 & AA & & \\
\hline Etoposide & GSTP1 & rs1695 & AA (lle/lle) & 3 & Decreased efficacy \\
\hline \multirow[t]{4}{*}{ Fluorouracil } & MTHFR & rs1801133 & $\pi$ & $2 \mathrm{~A}$ & Decreased efficacy \\
\hline & $D P Y D$ & rs3918290 & GG & $1 \mathrm{~A}$ & Reduced side-effects \\
\hline & & rs55886062 & $\pi$ & $1 \mathrm{~A}$ & \\
\hline & & rs67376798 & AA & $1 \mathrm{~A}$ & \\
\hline Irinotecan & UGT1A1 & rs4148323 & GG & $2 \mathrm{~A}$ & Reduced side-effects \\
\hline Pemetrexed & MTHFR & rs1801133 & $\pi$ & $2 \mathrm{~A}$ & Decreased efficacy \\
\hline \multirow[t]{2}{*}{ Vincristine/docetaxel/paclitaxel } & $A B C B 1$ & rs2032582 & GG & 3 & Decreased efficacy \\
\hline & CYP1B1 & rs1056836 & CC (Leu/Leu) & 3 & Increased efficacy \\
\hline
\end{tabular}




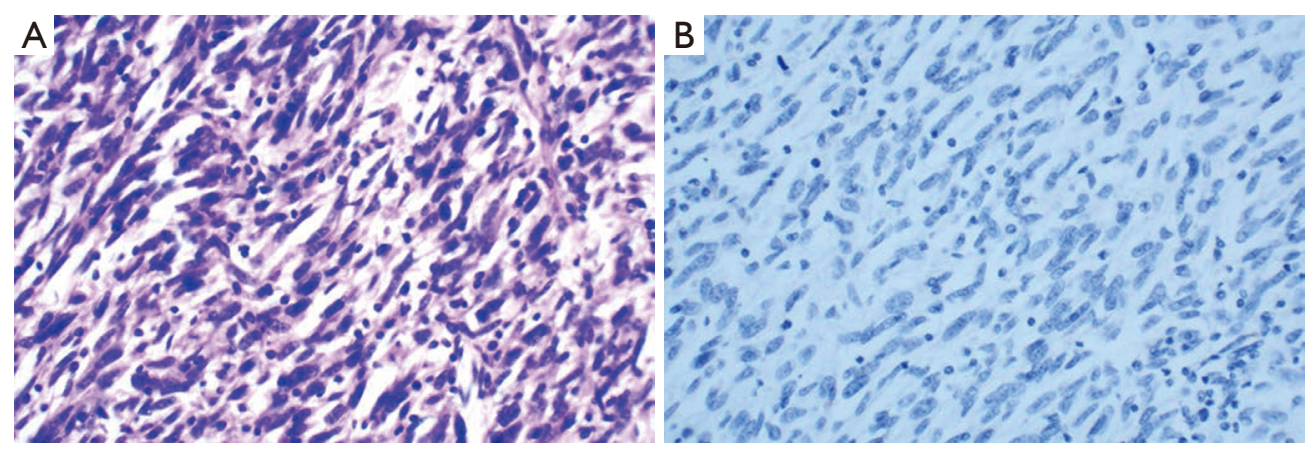

Figure 4 CD274 (programmed death-ligand 1 or PD-L1) level identification. (A) Hematoxylin-eosin (H-E) staining, $\times 400$. (B) PD-L1 antibody staining, $\times 400$.

Table 3 Timeline of the case

\begin{tabular}{ll}
\hline Time & Event \\
\hline Day 0 & Patient admission \\
Day 2 & Completion of preoperative examinations \\
Day 3 & Surgical resection of primary tumor \\
Day 7 & Patient discharge \\
11 months after surgery & Patient death \\
\hline
\end{tabular}

colonoscopy and tissue biopsy. CT scan can help evaluate tumor depth, regional lymph node status, and distant metastasis. Approximately $30 \%$ of diagnosed cases are found to have distant metastasis (7). Histological markers can be critical in the diagnosis of anorectal melanoma, in which S-100, human melanoma black (HMB-45), and vimentin are frequently positive. Microscopic findings of perineural invasion and depth of invasion are associated with poor prognosis $(8,9)$.

Multidisciplinary treatments are recommended to yield the best possible outcome, despite poor prognosis, among which surgical resection plays a main role. Complete resection with margins of more than $10 \mathrm{~mm}$ is associated with improved overall survival. There is no significant difference between wide local excision and abdominoperineal resection $(5,10)$.

Immunotherapies are increasingly found to be promising for the systemic treatment of advanced melanoma. The utilization of immunotherapy has increased in recent years. Some patients who received immunotherapy had significantly better 2 -year overall survival rates (11). Tumor cells employ immune evasion mechanisms in the processes of invasion and proliferation (12). Programmed cell death protein 1 (PD-1) on T cells can be bound with PD-L1 on tumor cells, limiting immune response. Specific antibodies against the receptor-ligand interaction remove the inhibition of T-cell activation, compromising the immune evasion of tumor cells (13).

There is little consensus on the optimal chemotherapy or radiation regimens for anorectal melanoma due to lack of convincing data from clinical trials (14). While the chemotherapy of anorectal melanoma can be carried out according to the chemotherapy scheme of cutaneous melanoma in some cases, chemotherapeutic agents are applied mostly in personalized settings. Commonly used agents include dacarbazine, temozolomide, paclitaxel, and carboplatin, which generally demonstrate modest response rates (15).

In summary, there is insufficient information on the treatment of anorectal melanoma, such as a consensus staging system, molecular classification, and adjuvant regimens. Screening tests are essential for the early diagnosis of anorectal melanoma. Timely surgical resection and subsequent multidisciplinary treatments are crucial for the long-term survival of the patients.

\section{Acknowledgments}

We feel grateful to the doctors and staff involved for their help.

Funding: None.

\section{Footnote}

Reporting Checklist: The authors have completed the CARE reporting checklist. Available at https://dx.doi. org/10.21037/apm-21-2240 
Conflicts of Interest: Both authors have completed the ICMJE uniform disclosure form (available at https://dx.doi. org/10.21037/apm-21-2240). The authors have no conflicts of interest to declare.

Ethical Statement: The authors are accountable for all aspects of the work in ensuring that questions related to the accuracy or integrity of any part of the work are appropriately investigated and resolved. All procedures performed in studies involving human participants were in accordance with the ethical standards of the institutional and/or national research committee(s) and with the Helsinki Declaration (as revised in 2013). Written informed consent was obtained from the patient for publication of this manuscript and any accompanying images. A copy of the written consent is available for review by the editorial office of this journal.

Open Access Statement: This is an Open Access article distributed in accordance with the Creative Commons Attribution-NonCommercial-NoDerivs 4.0 International License (CC BY-NC-ND 4.0), which permits the noncommercial replication and distribution of the article with the strict proviso that no changes or edits are made and the original work is properly cited (including links to both the formal publication through the relevant DOI and the license). See: https://creativecommons.org/licenses/by-nc-nd/4.0/.

\section{References}

1. Iddings DM, Fleisig AJ, Chen SL, et al. Practice patterns and outcomes for anorectal melanoma in the USA, reviewing three decades of treatment: is more extensive surgical resection beneficial in all patients? Ann Surg Oncol 2010;17:40-4.

2. Wild C. World cancer report 2014. Wild CP, Stewart BW, editors. Geneva, Switzerland: World Health Organization; 2014.

3. Yeh JJ, Shia J, Hwu WJ, et al. The role of abdominoperineal resection as surgical therapy for anorectal melanoma. Ann Surg 2006;244:1012-7.

Cite this article as: Cai X, Zhu X. Anorectal melanoma and gene analysis of personalized adjuvant therapy: a case report. Ann Palliat Med 2021;10(10):11216-11220. doi: 10.21037/apm-212240
4. Tacastacas JD, Bray J, Cohen YK, et al. Update on primary mucosal melanoma. J Am Acad Dermatol 2014;71:366-75.

5. Nilsson PJ, Ragnarsson-Olding BK. Importance of clear resection margins in anorectal malignant melanoma. Br J Surg 2010;97:98-103.

6. Singer M, Mutch MG. Anal melanoma. Clin Colon Rectal Surg 2006;19:78-87.

7. Stefanou A, Nalamati SP. Anorectal melanoma. Clin Colon Rectal Surg 2011;24:171-6.

8. Ishizone S, Koide N, Karasawa F, et al. Surgical treatment for anorectal malignant melanoma: report of five cases and review of 79 Japanese cases. Int J Colorectal Dis 2008;23:1257-62.

9. Yap LB, Neary P. A comparison of wide local excision with abdominoperineal resection in anorectal melanoma. Melanoma Res 2004;14:147-50.

10. Ragnarsson-Olding BK, Nilsson PJ, Olding LB, et al. Primary ano-rectal malignant melanomas within a population-based national patient series in Sweden during 40 years. Acta Oncol 2009;48:125-31.

11. Taylor JP, Stem M, Yu D, et al. Treatment Strategies and Survival Trends for Anorectal Melanoma: Is it Time for a Change? World J Surg 2019;43:1809-19.

12. Woo SR, Turnis ME, Goldberg MV, et al. Immune inhibitory molecules LAG-3 and PD-1 synergistically regulate $\mathrm{T}$-cell function to promote tumoral immune escape. Cancer Res 2012;72:917-27.

13. Wang C, Thudium KB, Han M, et al. In vitro characterization of the anti-PD-1 antibody nivolumab, BMS-936558, and in vivo toxicology in non-human primates. Cancer Immunol Res 2014;2:846-56.

14. Houghton AN, Coit DG, Daud A, et al. Melanoma. J Natl Compr Canc Netw 2006;4:666-84.

15. Eigentler TK, Caroli UM, Radny P, et al. Palliative therapy of disseminated malignant melanoma: a systematic review of 41 randomised clinical trials. Lancet Oncol 2003;4:748-59.

(English Language Editor: R. Scott) 